\title{
Prolyl Isomerase Pin1 Directly Regulates Calcium/Calmodulin- Dependent Protein Kinase II Activity in Mouse Brains
}

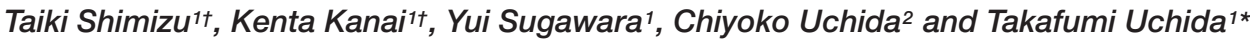 \\ 'Molecular Enzymology, Department of Molecular Cell Science, Graduate School of Agricultural Science, Tohoku University, \\ Sendai, Japan, ${ }^{2}$ Department of Human Development and Culture, Fukushima University, Fukushima, Japan
}

OPEN ACCESS

Edited by:

Tiziano Tuccinardi,

Università degli Studi di Pisa, Italy

Reviewed by:

Isabelle Landrieu,

UMR8576 Unité de Glycobiologie

Structurale et Fonctionnelle, France

Cordelia Schiene-Fischer

Martin Luther University

of Halle-Wittenberg, Germany

*Correspondence:

Takafumi Uchida

uchidataka@gmail.com

tThese authors have contributed

equally to this work

Specialty section:

This article was submitted to

Experimental Pharmacology

and Drug Discovery,

a section of the journal

Frontiers in Pharmacology

Received: 06 September 2018

Accepted: 05 November 2018

Published: 22 November 2018

Citation:

Shimizu T, Kanai K, Sugawara $Y$, Uchida C and Uchida T (2018) Prolyl Isomerase Pin1 Directly Regulates Calcium/Calmodulin-Dependent

Protein Kinase II Activity in Mouse Brains. Front. Pharmacol. 9:1351.

doi: 10.3389/fphar.2018.01351
Calcium/calmodulin-dependent protein kinase II (CaMKII) is abundant in the brain and functions as a mediator of calcium signaling. We found that the relative activity of CaMKII was significantly lower in the WT mouse brains than in the Pin1-/- mouse brains. Pin1 binds to phosphorylated CaMKII and weakens its activity. For this reason, the phosphorylation level of tau in the presence of Pin1 is lower than that in the absence of Pin1, and microtubule polymerization is not downregulated by CaMKII when Pin1 is present. These results suggest a novel mechanism of action of Pin1 to prevent neurodegeneration.

Keywords: prolyl isomerase, Pin1, tau, CaMKII, tauopathy

\section{INTRODUCTION}

The peptidyl prolyl cis/trans isomerase Pin1 binds to phosphorylated Ser/Thr-Pro and isomerizes the peptide bond. Pin $1^{-/-}$mice (Fujimori et al., 1999) had Alzheimer's disease-like phenotypes (Liou et al., 2003; Xu et al., 2017). However, the relationship between Pin 1 and Alzheimer's disease remains unclear (Akiyama et al., 2005; Pastorino et al., 2006). We have previously performed GSTPin1 pull down analysis using mouse brains to determine the proteins that interact with Pin1 in the brain, and we identified several proteins including histone, synapsin, and CaMKII (Tatara et al., 2010). We speculate that Pin 1 controls various neuronal functions associated with the CaMKII signaling pathway.

Calcium/calmodulin-dependent protein kinase II is abundant in the brain. It functions as a mediator of calcium signaling, and plays an important role in synaptic plasticity, which affects learning and memory (Lisman et al., 2002). It has been reported that the overexpression of CaMKII inhibits neuronal differentiation in PC12 cells (Massé and Kelly, 1997), and that constitutive CaMKII activation impairs the development of cholinergic synapses in giant fibers (Kadas et al., 2012). Dysregulation of CaMKII has been observed in neurological diseases including ADHD (Yabuki et al., 2014), Parkinson's disease (Zaichick et al., 2017), and Alzheimer's disease (Ghosh and Giese, 2015). These reports suggested that adequately regulating CaMKII in the brain is important for preventing neuronal dysfunction.

One of the substrates of CaMKII is tau, a microtubule-associated protein that presents mainly on axons in neuronal cells and promotes microtubule polymerization (Drechsel et al., 1992).

Abbreviations: ADHD, attention deficit hyperactivity disorder; CaMKII, calcium/calmodulin-dependent protein kinase II; N2a, Neuro2a; $P$ in $1^{-/-}$mice, mice lacking Pin1 gene; WT mice, wild type mice. 
The function and stability of tau are regulated by several kinds of modifications, such as acetylation, glycation, ubiquitination, and phosphorylation (Mietelska-Porowska et al., 2014). Phosphorylation is the most important modification that regulates tau function. In general, phosphorylation decreases tau activity (Johnson and Stoothoff, 2004). Proper regulation of tau phosphorylation is very important for microtubule control; the accumulation of hyperphosphorylated tau aggregates and neuronal loss are observed in the brains of tauopathy patients (Šimić et al., 2016).

In the present paper, we showed that Pin1 binds to CaMKII directly and weakens its relative activity, which reduces the level of phosphorylated tau. When there is a high level of tau phosphorylation, microtubule polymerization is not regulated properly. These results suggest a novel mechanism of action of Pin 1 to prevent neurodegeneration.

\section{EXPERIMENTAL PROCEDURES}

\section{Animals}

Pin $1^{-/-}$mice were generated and bred according to the methods described in our previous report (Fujimori et al., 1999). Our study was approved by the Tohoku University Animal Use and Care Committee, and all investigations were conducted according to the principles of the Declaration of Helsinki. Genotypes of the mice bred by mating Pin $1^{+/-}$mice were examined using the polymerase chain reaction (PCR). Primers: WILD1.2A (5-AAG GGA TTA GAA GCA AGA TTC G-3), 2L (5-AGC ACC CGA TCC TGT TCT GCA A-3'), and Start2 (5-CAG AGG CCA CTT GTG TA-3') (Fujimori et al., 1999).

\section{Cell Culture}

Neuro2a (N2a) cells were cultured in Dulbecco's modified Eagle's medium (DMEM; Nacalai Tesque Inc.) supplemented with $10 \%$ fetal bovine serum (FBS) and $1 \%$ penicillin/streptomycin. The cells were grown in $5 \% \mathrm{CO}_{2}$ at $37^{\circ} \mathrm{C}$, and transfected with the following plasmids as previously demonstrated (Shimizu et al., 2016): Pin1 WT, Pin1 mutant W34A (WW mutant: Pin1 without WW domain function), R68/69A (PPIase mutant: Pin1 without substrate affinity), and flag-CaMKII $\alpha$. Transfection was performed using Lipofectamine 2000 (Qiagen).

\section{Western Blotting}

The cells were lysed in sample buffer (62.5 mM Tris$\mathrm{HCl}$, 5\% sucrose, 2\% sodium dodecyl sulfate (SDS), 5\% $\beta$-mercaptoethanol, $\mathrm{pH}$ 6.8). The samples were analyzed by sodium dodecyl sulfate-polyacrylamide gel electrophoresis and western blotting using the following antibodies: antiCaMKII antibody (1:1000) (H-300; Santa Cruz), anti-Pin1 antibody (1:2000) (Cell Signaling Technology, Danvers, MA, United States), Phospho-Tau Ser416 antibody (1:1000) (Cell Signaling Technology), Phospho-Tau Ser262 antibody (1:2000) (Thermo Fisher Scientific), and Tau5 antibody (1:2000) (BD Biosciences) for total tau. LAS-3000 (Fujifilm) was used for detection. MultiGauge software (Fujifilm) was used to measure the bands semi-quantitatively.

\section{CaMKII Kinase Assay}

Mouse brains were lysed in extraction buffer (100 mM piperazine- $\mathrm{N}, \mathrm{N}^{\prime}$-bis(2-ethanesulfonic acid) (PIPES), pH 6.9; $10 \mathrm{mM}$ ethylenediamine tetraacetic acid (EDTA); $10 \mathrm{mM}$ ethylene glycol-bis $(\beta$-aminoethyl ether $)-\mathrm{N}, \mathrm{N}, \mathrm{N}^{\prime}, \mathrm{N}^{\prime}$-tetraacetic acid (EGTA); $1 \mathrm{mM}$ phenylmethylsulfonyl fluoride (PMSF); $2 \mathrm{mM} \mathrm{Na}_{3} \mathrm{VO}_{4} ; 2 \mathrm{mM} \mathrm{NaF} ; 2 \mathrm{mM}$ dithiothreitol (DTT); protease inhibitor cocktail (Nacalai Tesque Inc.). The N2a cells were transiently transfected with the flag-CaMKII $\alpha$ and Pin1 (WT, $W 34 A, R 68 / 69 A$ ) plasmids. After 24-48 h, the cells were lysed in the extraction buffer. The cell lysates were analyzed using a CaMKII Assay Kit (Promega). The substrate peptide of this kit contains no Ser/Thr-Pro site to which Pin1 can bind. ATP- $\gamma$-32P (3000 Ci/mmol, $10 \mathrm{mCi} / \mathrm{mL}$; PerkinElmer). The expression level of the CaMKII proteins was measured semi-quantitatively from the intensities of western blot bands using MultiGauge software (Fujifilm).

The relative activity (kinase activity/protein level) was determined from these experimental results. Recombinant CaMKII $\alpha$ derived from baculovirus (Carna Biosciences Inc.) was incubated with recombinant Pin 1 for $1 \mathrm{~h}$. The activity was determined using a CaMKII Assay Kit (Promega) with ATP$\gamma^{-32} \mathrm{P}(3000 \mathrm{Ci} / \mathrm{mmol}, 10 \mathrm{mCi} / \mathrm{mL}$; PerkinElmer) or a western blotting investigation of tau phosphorylation at Ser262.

\section{Microtubule Polymerization Assay}

$10 \mu \mathrm{M}$ of tau was phosphorylated by $10 \mathrm{nM}$ CaMKII $\alpha$ with or without $40 \mathrm{nM}$ Pin 1 for $3 \mathrm{~h}$ or phosphorylated by $50 \mathrm{nM}$ CaMKII $\alpha$ with or without $100 \mathrm{nM}$ Pin 1 for $14.5 \mathrm{~h}$. These samples were directly added to the microtubule polymerization assay mixtures (Uchida et al., 2009; Gotoh et al., 2013). The optical density of the polymerized microtubules solution was measured at $350 \mathrm{~nm}\left(A_{350}\right)$.

\section{Statistical Analysis}

Results were reported as mean \pm SD. Statistical significance was determined by Student's $t$ test or Dunnett's test (multiple test). We evaluated that the value of $p<0.05$ was statistically significant.

\section{RESULTS}

\section{Comparison of CaMKII Activity, Protein Levels, and Specific Activity Between Wild-Type (WT) and Pin1-/- Mouse Brains}

We compared CaMKII kinase activity and CaMKII expression levels among brain lysates of WT and Pin $1^{-/-}$mice $(n=12$ and 13 mice, respectively). Because of numerical variations between mice, there was not significant difference between them but the total CaMKII kinase activity in the Pin $1^{-/-}$mouse brains tended to be higher than that in the WT mouse brains (Figure 1A). The CaMKII expression levels did not differ significantly between the two (Figures 1B,C). The values obtained from these analyses are summarized in Figure 1D. The specific CaMKII activities (total kinase activity/protein level) were calculated and compared 

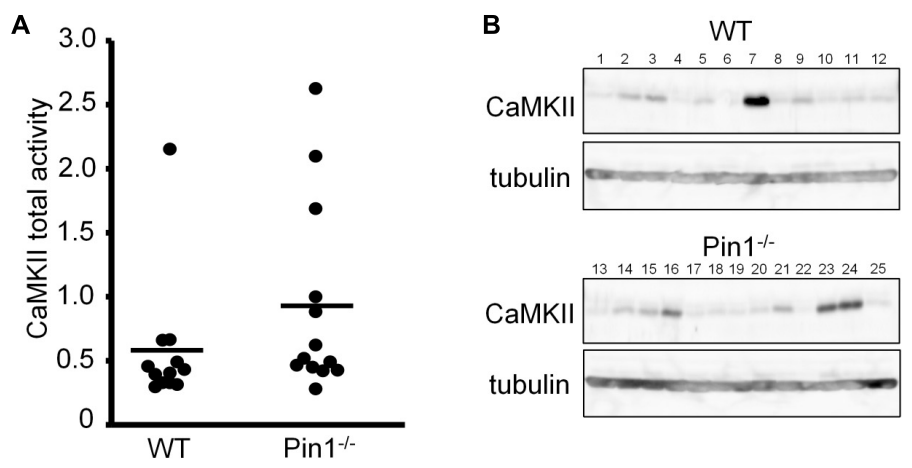

D

WT

\begin{tabular}{|l|c|c|c|c|c|c|c|c|c|c|c|c|}
\hline Sample No. & 1 & 2 & 3 & 4 & 5 & 6 & 7 & 8 & 9 & 10 & 11 & 12 \\
\hline total activity & 0.30 & 0.66 & 0.66 & 0.33 & 0.33 & 0.32 & 2.15 & 0.40 & 0.49 & 0.40 & 0.43 & 0.46 \\
\hline protein level & 0.13 & 0.37 & 0.47 & 0.12 & 0.20 & 0.09 & 2.60 & 0.30 & 0.39 & 0.16 & 0.25 & 0.23 \\
\hline Pin $1^{-1-}$ \\
\begin{tabular}{|c|c|c|c|c|c|c|c|c|c|c|c|c|c|}
\hline Sample No. & 13 & 14 & 15 & 16 & 17 & 18 & 19 & 20 & 21 & 22 & 23 & 24 & 25 \\
\hline total activity & 0.28 & 0.62 & 0.88 & 1.69 & 0.42 & 0.49 & 0.52 & 0.47 & 1.00 & 0.43 & 2.10 & 2.63 & 0.45 \\
\hline protein level & 0.15 & 0.28 & 0.37 & 0.80 & 0.13 & 0.14 & 0.11 & 0.17 & 0.37 & 0.09 & 1.00 & 1.26 & 0.11 \\
\hline
\end{tabular}
\end{tabular}

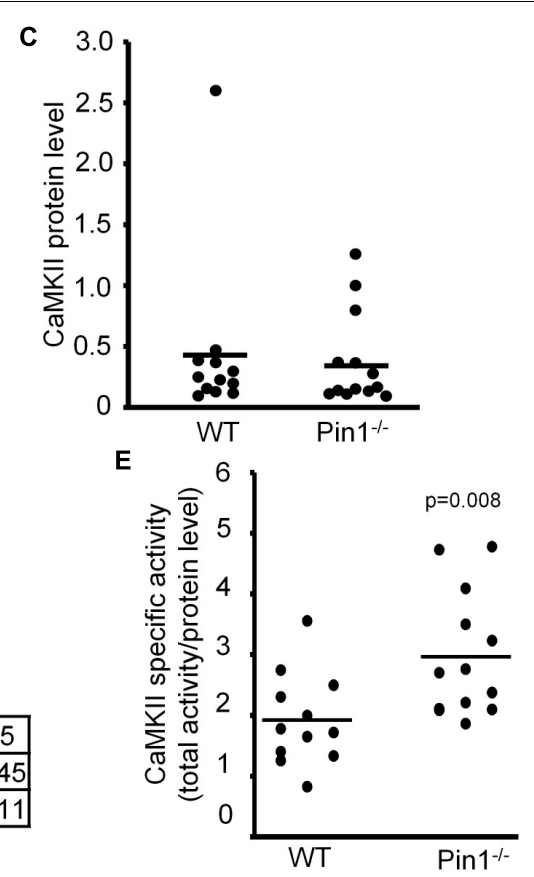

FIGURE 1 | Activity and expression levels of CaMKII in wild-type (WT) and Pin $1^{-/-}$mouse brains. (A) CaMKII total activities in WT and Pin $1^{-/-}$mouse brain lysates ( $n=12$ and 13 distinct mouse brains, respectively) were analyzed using a CaMKII Assay Kit with ATP- $\gamma-32$, and the relative values were plotted. (B) Western blot analysis of CaMKII. Tubulin was used as a loading control. (C) Relative expression levels of CaMKII in the brain lysates of WT and Pin $1^{-/-}$mice. The western bands were measured semi-quantitatively from the intensities of western blot bands using MutiGauge software. $p=0.8$ using the Student's $t$-test (not significant difference). (D) Relative values of CaMKII total activities and protein levels in WT and Pin $1^{-/-}$mouse brain lysates. (E) Each CaMKII-specific activity in the WT and Pin $1^{-/-}$ mouse brain lysates was estimated by dividing the relative value of the total activity by the relative value of the protein level. $p=0.008$ using the Student's $t$-test.

between the WT and Pin1 $1^{-/-}$mouse brains. The specific activity of CaMKII was significantly lower $(p=0.008)$ in the WT brains than in the Pin $1^{-/-}$brains (Figure 1E).

\section{Comparison of Phosphorylated Tau Between WT and Pin1-/- Mouse Brains}

It has previously been reported that Ser262 of tau is phosphorylated by CaMKII and it has been reported to be associated with Alzheimer's disease (Seubert et al., 1995; Singh et al., 1996). In addition to Ser262, it has also been reported Ser416 is phosphorylated by CaMKII (Yamamoto et al., 2005). First, it was confirmed that the levels of phosphorylated Ser262 tau and phosphorylated Ser416 tau were increased in the N2a cells transfected with CaMKIIa cDNA (Figure 2A). Next, the phosphorylated Ser262 and phosphorylated Ser416 tau levels in the brain lysates from WT and Pin $1^{-/-}$mice $(n=3$ mice, respectively) were compared. Phosphorylated Ser262 was not detected in either of brains, but the phosphorylated Ser416 was detected (Figure 2B). The value of phosphorylated Ser416 tau/ total tau was significantly higher in the Pin $1^{-/-}$mouse brains than in the WT mouse brains (Figures 2B,C). These results indicate that Pin1 regulates CaMKII activity in mouse brains.

\section{Pin1 Suppressed CaMKII Activity}

We examined the effect of Pin1 on CaMKII total activity in N2a cells. N2a cells transfected with flag-CaMKIIa, Pin1 WT, W34A,

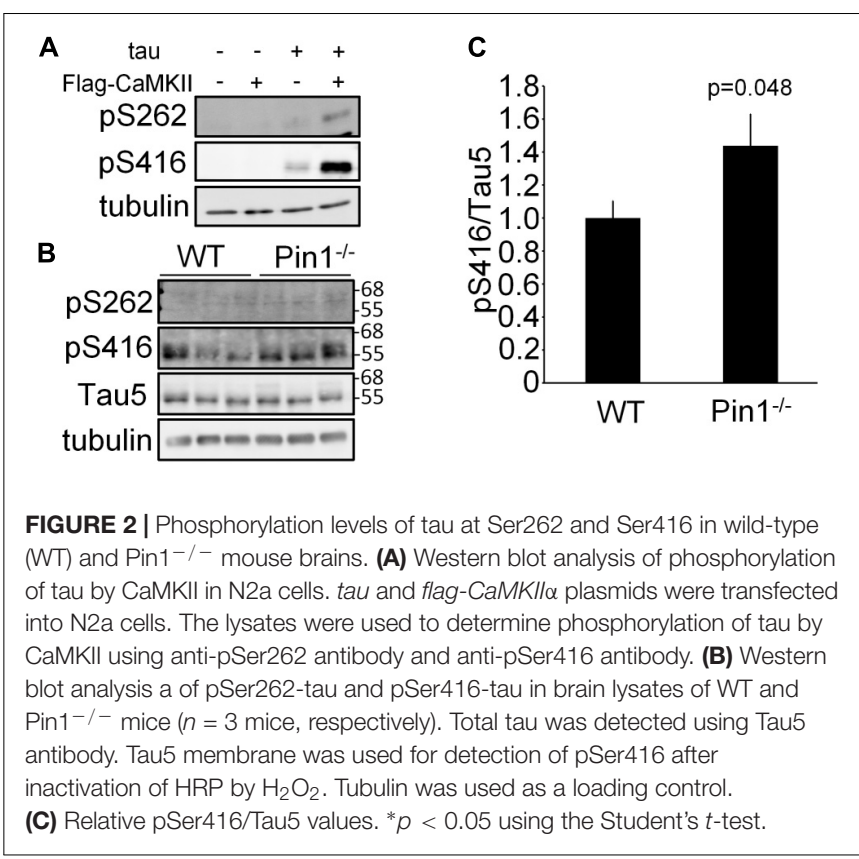

and $R 68 / 69 A$ plasmids were analyzed using a CaMKII kinase assay (Figure 3A). The co-expression of the Pin1 WT markedly reduced CaMKII total activity. The co-expression of the Pin1 mutant at the WW domain (Pin1 W34A) did not significantly 
A

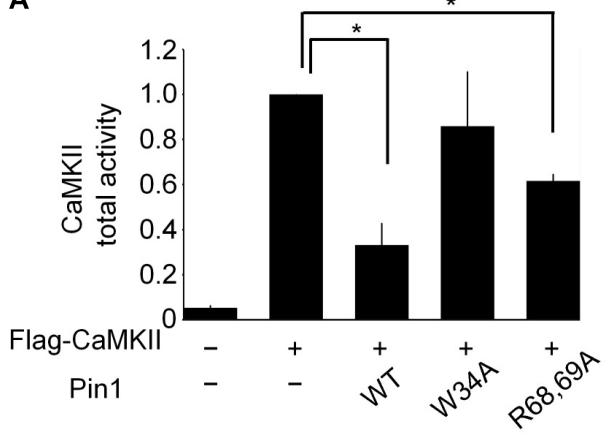

B

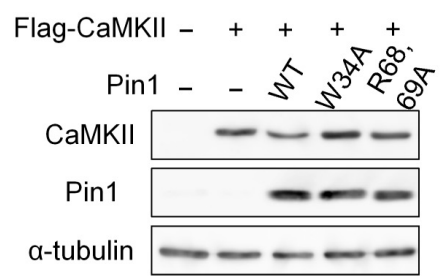

C

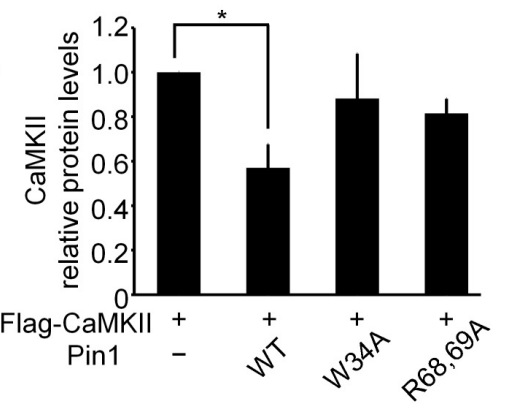

FIGURE 3 | CaMKII activity and expression levels in Neuro2a cells. (A) CaMKII total activity in N2a cells. A flag-CaMKIla plasmid was transfected with mock or pin1 plasmids into N2a cells. The lysates were used to determine CaMKIl total activity. The data represent the means \pm SDs of three independent experiments. ${ }^{*} p<0.05$ using Dunnett's test. (B) Protein levels of CaMKII in N2a cells were analyzed by western blotting after transfection of the flag-CaMKIla plasmid with mock or pin1 plasmids. Tubulin was used as a loading control. (C) Relative values of CaMKIl protein levels in panel (B). The data represent means \pm SDs of three independent experiments. ${ }^{*} p<0.05$ using Dunnett's test.

reduce CaMKII activity. Pin1 mutant activity at the PPIase domain (Pin1 R68/69A) decreased even though suppression was weaker than in the WT. These results suggest that Pin 1 suppresses CaMKII total activity, and that both the WW domain and the PPIase domain are required for this effect. Although the expression level of CaMKII was also reduced by Pin 1 in the N2a cells (Figures 3B,C), the effect was not as pronounced as the effect on total activity. These results indicate that Pin 1 suppresses CaMKII activity in N2a cells.

\section{Pin1 Reduced the Phosphorylation of Tau by CaMKII}

To determine whether Pin1 suppresses CaMKII-specific activity, we performed a CaMKII kinase assay in vitro using baculovirusderived recombinant CaMKII $\alpha$. CaMKII activity was reduced significantly by Pin1 (Figure 4A). We speculated whether Pin1 regulates the phosphorylation of tau by CaMKII. Recombinant tau was incubated with CaMKII with or without Pin1, and each phosphorylated tau was examined by western blotting using anti-tau pS262 antibody. As expected, Pin1 reduced the phosphorylation level of Ser262 of tau during at least $180 \mathrm{~min}$ (Figure 4B). It has been reported that phosphorylation of Ser262 reduces the interaction between tau and microtubules (Biernat et al., 1993). However, the microtubule polymerization activities of the three different kinds of tau prepared by the incubation with tau only, tau plus CaMKII, and tau plus CaMKII and Pin1, for $180 \mathrm{~min}$ were not different (Figure 4C). These results suggest that phosphorylation at Ser 262 is insufficient for tau to reduce microtubule polymerization as previously reported (Singh et al., 1996).

\section{Pin1 Rescued the Microtubule Polymerization Activity of CaMKII-Phosphorylated Tau}

We performed an in vitro microtubule polymerization assay using tau phosphorylated by CaMKII in the presence and absence

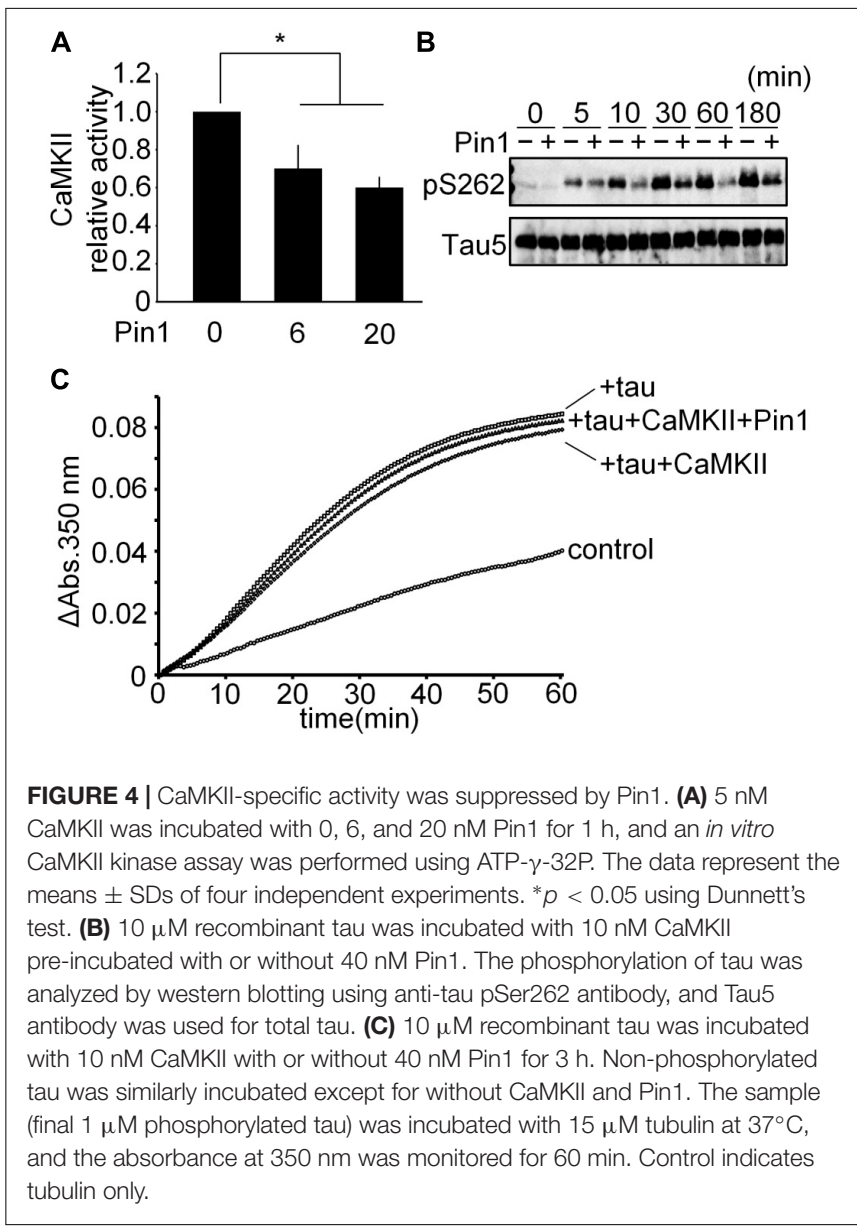

of Pin 1 for $14.5 \mathrm{~h}$. Although presence of Pin 1 did not change the phosphorylation levels of Ser262 of these samples (Figure 5A), the sample containing CaMKII-phosphorylated tau in the presence of Pin1 exhibited higher microtubule polymerization 


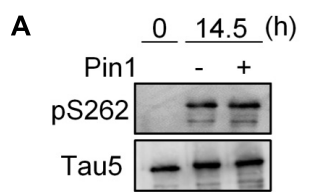

B

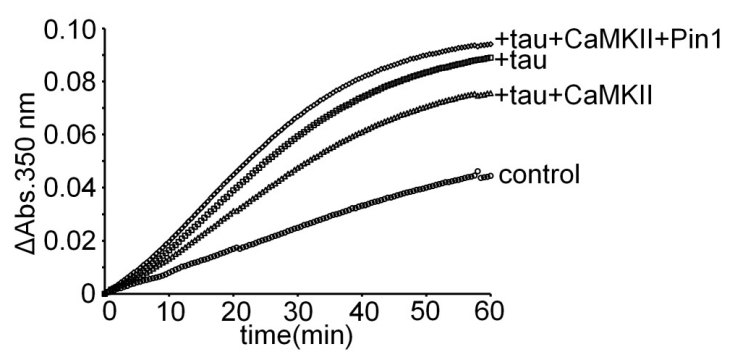

FIGURE 5 | Pin1 reduced the effect of CaMKII on tau microtubule polymerization activity. (A) $10 \mu \mathrm{M}$ recombinant tau was incubated with $50 \mathrm{nM}$ CaMKII with or without 100 nM Pin1 for 14.5 h. Phosphorylated Ser262 tau and total tau were analyzed by western blotting using anti-tau pSer262 antibody and Tau5 antibody. (B) $10 \mu \mathrm{M}$ recombinant tau was incubated with $50 \mathrm{nM}$ CaMKII with or without 100 nM Pin1 for $14.5 \mathrm{~h}$. Non-phosphorylated tau was similarly incubated except for without CaMKII and Pin1. The sample (final $1 \mu \mathrm{M}$ phosphorylated tau) was incubated with $15 \mu \mathrm{M}$ tubulin at $37^{\circ} \mathrm{C}$, and the absorbance at $350 \mathrm{~nm}$ was monitored for $60 \mathrm{~min}$. Three independent experiments were performed. Control indicates tubulin only.

activity than that in the absence of Pin 1 (Figure 5B). These results suggest that Pin 1 enhances the activity of tau phosphorylated by CaMKII.

\section{DISCUSSION}

We have shown that Pin 1 binds to CaMKII in the mouse brain (Tatara et al., 2010), but Ser/Thr-Pro site that phosphorylated by CaMKII has not been identified. CaMKII has four Ser/ThrPro sites, while Ser234 is the only phosphorylation site (Trinidad et al., 2008). Therefore, we speculate that Pin1 binds to phosphorylated Ser234-Pro in CaMKII. It is known that Pin1 binds to CaMKII, but it is not known the function of Pin1 for CaMKII. Since Pin1 decreases the activity of CaMKII, we speculated that Pin1 might decrease the amount of CaMKII or decrease its relative activity. By comparing the amount and activity of CaMKII in the brains of Pin $1^{-/-}$and WT mice, we found that the relative activity of CaMKII is suppressed by Pin1.

The dysregulation of signal transduction in the brain causes various neuronal functional disorders related to movement, language, and memory. Tauopathies and Alzheimer's disease are caused by aberrant tau (Lee et al., 2001). Phosphorylation is closely related to aberration of tau and CaMKII also phosphorylates some functional sites of tau including phosphorylates Ser262 in the repeat domain of tau (Yoshimura et al., 2003), which reduces the affinity of tau to a microtubule (Biernat et al., 1993). These findings suggest that the activity of CaMKII profoundly affects the function of tau, although it has not been elucidated yet which phosphorylation site is the most important for tau activity.
Ser262 of tau was highly phosphorylated within $30 \mathrm{~min}$. However, this phosphorylation did not decrease the microtubule polymerization activity of tau. On the other hand, the tau prepared by incubating with CaMKII for about $15 \mathrm{~h}$ reduced the activity of tau. These results show that phosphorylation of Ser262 is insufficient to decrease the affinity to microtubules as previously reported (Singh et al., 1996). We speculate that there are the other sites that are phosphorylated more slowly than Ser262 by CaMKII in vitro. It has been reported that Pin1 promotes the dephosphorylation of tau (Landrieu et al., 2011) and stabilizes microtubules via phosphorylated tau ( $\mathrm{Lu}$ et al., 1999; Shimizu et al., 2017). Pin $1^{-/-}$mice had aberrant tau and degenerated axons in brains (Liou et al., 2003). However, the molecular mechanism behind the effect of Pin 1 on microtubule polymerization is still controversial (Kutter et al., 2016). In our experiments, phosphorylation of tau was performed by CaMKII that is different from the kinase they used. Although we do not exclude the direct effect of Pin1 on the specific phosphorylated Ser/Thr-Pro site, we suspect that the total phosphorylation level of tau might induce the change of tau.

Calcium/calmodulin-dependent protein kinase II plays an important role in memory because it phosphorylates various substrates including the neurotransmission-related protein Synapsin I (Benfenati et al., 1992), AMPAR (Hayashi et al., 2000), and NMDAR (Sanhueza et al., 2011), as well as tau. $\mathrm{CaMKII}^{-/-}$mice exhibit specific learning impairments (Silva et al., 1992), and aberrant CaMKII activity is associated with cognitive dysfunction in ADHD model rats (Yabuki et al., 2014). It has also been reported that Pin $1^{-/}$mice have significantly enhanced late longterm potentiation (LTP) compared to WT mice (Westmark et al., 2010). Therefore, we speculate that Pin1 might play an important role in memory via $\mathrm{Ca}^{2+}$ signal pathways as well as neurological diseases like tauopathy and Alzheimer's disease. We speculate that Pin1 might control many brain diseases by regulating CaMKII activity properly. In this report, we showed a novel mechanism of action of Pin 1 to prevent neurodegeneration.

\section{AUTHOR CONTRIBUTIONS}

TU conceived the study and administered the overall study. TU and CU provided resources. TS, KK, and YS performed the experiments. TS and TU analyzed the data and wrote the manuscript.

\section{FUNDING}

TU was supported by a Grant-in-Aid for Scientific Research from the Ministry of Education, Culture, Sports, Science, and Technology of Japan (Kiban A; 26252064, Kiban S;20228006). The funders had no role in study design, data collection and analysis, decision to publish, or preparation of the manuscript. 


\section{REFERENCES}

Akiyama, H., Shin, R.-W., Uchida, C., Kitamoto, T., and Uchida, T. (2005). Pin 1 promotes production of Alzheimer's amyloid $\beta$ from $\beta$-cleaved amyloid precursor protein. Biochem. Biophys. Res. Commun. 336, 521-529. doi: 10.1016/ j.bbrc.2005.08.130

Benfenati, F., Valtorta, F., Rubenstein, J. L., Gorelick, F. S., Greengard, P., and Czernik, A. J. (1992). Synaptic vesicle-associated Ca2+/calmodulin-dependent protein kinase II is a binding protein for synapsin I. Nature 359, 417-420. doi: $10.1038 / 359417 \mathrm{a} 0$

Biernat, J., Gustke, N., Drewes, G., Mandelkow, E., and Mandelkow, E. (1993). Phosphorylation of Ser262 strongly reduces binding of tau to microtubules: distinction between PHF-like immunoreactivity and microtubule binding. Neuron 11, 153-163. doi: 10.1016/0896-6273(93)90279-Z

Drechsel, D. N., Hyman, A. A., Cobb, M. H., and Kirschner, M. W. (1992). Modulation of the dynamic instability of tubulin assembly by the microtubuleassociated protein tau. Mol. Biol. Cell 3, 1141-1154. doi: 10.1091/mbc.3.10. 1141

Fujimori, F., Takahashi, K., Uchida, C., and Uchida, T. (1999). Mice lacking Pin1 develop normally, but are defective in entering cell cycle from $\mathrm{G}(0)$ arrest. Biochem. Biophys. Res. Commun. 265, 658-663. doi: 10.1006/bbrc.1999.1736

Ghosh, A., and Giese, K. P. (2015). Calcium/calmodulin-dependent kinase II and Alzheimer's disease. Mol. Brain 8:78. doi: 10.1186/s13041-015-0166-2

Gotoh, A., Hidaka, M., Hirose, K., and Uchida, T. (2013). Gas7b (growth arrest specific protein $7 \mathrm{~b}$ ) regulates neuronal cell morphology by enhancing microtubule and actin filament assembly. J. Biol. Chem. 288, 34699-34706. doi: 10.1074/jbc.M113.513119

Hayashi, Y., Shi, S. H., Esteban, J. A., Piccini, A., Poncer, J. C., and Malinow, R. (2000). Driving AMPA receptors into synapses by LTP and CaMKII: requirement for GluR1 and PDZ domain interaction. Science 287, 2262-2267. doi: $10.1126 /$ science.287.5461.2262

Johnson, G. V. W., and Stoothoff, W. H. (2004). Tau phosphorylation in neuronal cell function and dysfunction. J. Cell Sci. 117, 5721-5729. doi: 10.1242/jcs.01558

Kadas, D., Tzortzopoulos, A., Skoulakis, E. M. C., and Consoulas, C. (2012). Constitutive activation of $\mathrm{Ca} 2+/$ Calmodulin-dependent protein kinase II during development impairs central cholinergic transmission in a circuit underlying escape behavior in Drosophila. J. Neurosci. 32, 170-182. doi: 10. 1523/JNEUROSCI.6583-10.2012

Kutter, S., Eichner, T., Deaconescu, M. A., and Kern, D. (2016). Regulation of microtubule assembly by Tau and not by Pin1. J. Mol. Biol. 428, 1742-1759. doi: 10.1016/j.jmb.2016.03.010

Landrieu, I., Smet-Nocca, C., Amniai, L., Louis, J. V., Wieruszeski, J.-M., Goris, J., et al. (2011). Molecular implication of PP2A and Pin1 in the Alzheimer's disease specific hyperphosphorylation of Tau. PLoS One 6:e21521. doi: 10.1371/journal. pone. 0021521

Lee, V. M.-Y., Goedert, M., and Trojanowski, J. Q. (2001). Neurodegenerative tauopathies. Annu. Rev. Neurosci. 24, 1121-1159. doi: 10.1146/annurev.neuro. 24.1.1121

Liou, Y., Sun, A., Ryo, A., Zhou, X., Yu, Z., Huang, H., et al. (2003). Role of the prolyl isomerase Pin1 in protecting against age-dependent neurodegeneration. Nature 424, 556-561. doi: 10.1038/nature01834.1

Lisman, J., Schulman, H., and Cline, H. (2002). The molecular basis of CaMKII function in synaptic and behavioural memory. Nat. Rev. Neurosci. 3, 175-190. doi: $10.1038 / \mathrm{nrn} 753$

Lu, P. J., Wulf, G., Zhou, X. Z., Davies, P., and Lu, K. P. (1999). The prolyl isomerase Pin1 restores the function of Alzheimer-associated phosphorylated tau protein. Nature 399, 784-788. doi: 10.1038/21650

Massé, T., and Kelly, P. T. (1997). Overexpression of Ca2+/Calmodulin-dependent protein kinase II in PC12 Cells alters cell growth, morphology, and nerve growth factor-induced differentiation. J. Neurosci. 17, 924-931. doi: 10.1523/ JNEUROSCI.17-03-00924.1997

Mietelska-Porowska, A., Wasik, U., Goras, M., Filipek, A., and Niewiadomska, G. (2014). Tau protein modifications and interactions: their role in function and dysfunction. Int. J. Mol. Sci. 15, 4671-4713. doi: 10.3390/ijms15034671

Pastorino, L., Sun, A., Lu, P.-J., Zhou, X. Z., Balastik, M., Finn, G., et al. (2006). The prolyl isomerase Pin1 regulates amyloid precursor protein processing and amyloid- $\beta$ production. Nature $440,528-534$. doi: 10.1038/nature04543

Sanhueza, M., Fernandez-Villalobos, G., Stein, I. S., Kasumova, G., Zhang, P., Bayer, K. U., et al. (2011). Role of the CaMKII/NMDA Receptor Complex in the maintenance of synaptic strength. J. Neurosci. 31, 9170-9178. doi: 10.1523/ JNEUROSCI.1250-11.2011

Seubert, P., Mawal-Dewan, M., Barbour, R., Jakes, R., Goedert, M., Johnson, V. W. G., et al. (1995). Detection of phosphorylated Ser262 in fetal tau, adult tau, and paired helical filament tau. J. Biol. Chem. 270, 18917-18922. doi: 10.1074/JBC.270.32.18917

Shimizu, T., Bamba, Y., Kawabe, Y., Fukuda, T., Fujimori, F., Takahashi, K., et al. (2016). Prolyl isomerase Pin1 regulates doxorubicin-inducible P-glycoprotein level by reducing Foxo3 stability. Biochem. Biophys. Res. Commun. 471, 328333. doi: 10.1016/j.bbrc.2016.02.014

Shimizu, T., Uchida, C., Shimizu, R., Motohashi, H., and Uchida, T. (2017). Prolyl isomerase Pin1 promotes proplatelet formation of megakaryocytes via tau. Biochem. Biophys. Res. Commun. 493, 946-951. doi: 10.1016/j.bbrc.2017.09.115

Silva, A. J., Paylor, R., Wehner, J. M., and Tonegawa, S. (1992). Impaired spatial learning in alpha-calcium-calmodulin kinase II mutant mice. Science 257, 206-211. doi: 10.1126/science.1321493

Šimić, G., Babić Leko, M., Wray, S., Harrington, C., Delalle, I., JovanovMilošević, N., et al. (2016). Tau Protein hyperphosphorylation and aggregation in Alzheimer's disease and other tauopathies, and possible neuroprotective strategies. Biomolecules 6:6. doi: 10.3390/biom6010006

Singh, J., Wang, J., Novak, M., Kontzekova, E., Grundke-Iqbal, I., and Iqbal, K. (1996). Calcium/calmodulin-dependent protein kinase II phosphorylates tau at Ser-262 but only partially inhibits its binding to microtubules. FEBS Lett. 387, 145-148. doi: 10.1016/0014-5793(96)00485-1

Tatara, Y., Terakawa, T., and Uchida, T. (2010). Identification of Pin1-binding phosphorylated proteins in the mouse brain. Biosci. Biotechnol. Biochem. 74, 2480-2483. doi: 10.1271/bbb. 100580

Trinidad, C. J., Thalhammer, A., Specht, G. C., Lynn, J. A., Baker, R. P., Schoepfer, R., et al. (2008). Quantitative analysis of synaptic phosphorylation and protein expression. Mol. Cell. Proteomics 7, 684-696. doi: 10.1074/mcp. M700170-MCP200

Uchida, T., Akiyama, H., Sakamoto, W., Koga, T., Yan, K., Uchida, C., et al. (2009). Direct optical microscopic observation of the microtubule polymerization intermediate sheet structure in the presence of gas7. J. Mol. Biol. 391, 849-857. doi: 10.1016/j.jmb.2009.06.081

Westmark, P. R., Westmark, C. J., Wang, S., Levenson, J., O'Riordan, K. J., Burger, C., et al. (2010). Pin1 and PKMzeta sequentially control dendritic protein synthesis. Sci. Signal. 3, ra18. doi: 10.1126/scisignal.2000451

Xu, L., Ren, Z., Chow, F. E., Tsai, R., Liu, T., Rizzolio, F., et al. (2017). Pathological Role of Peptidyl-Prolyl isomerase Pin1 in the disruption of synaptic plasticity in Alzheimer's disease. Neural Plast. 2017:3270725. doi: 10.1155/2017/3270725

Yabuki, Y., Shioda, N., Maeda, T., Hiraide, S., Togashi, H., and Fukunaga, K. (2014). Aberrant CaMKII activity in the medial prefrontal cortex is associated with cognitive dysfunction in ADHD model rats. Brain Res. 1557, 90-100. doi: 10.1016/j.brainres.2014.02.025

Yamamoto, H., Hiragami, Y., Murayama, M., Ishizuka, K., Kawahara, M., and Takashima, A. (2005). Phosphorylation of tau at serine416 by $\mathrm{Ca}$ $2+$ /calmodulin-dependent protein kinase II in neuronal soma in brain. J. Neurochem. 94, 1438-1447. doi: 10.1111/j.1471-4159.2005.03307.x

Yoshimura, Y., Ichinose, T., and Yamauchi, T. (2003). Phosphorylation of tau protein to sites found in Alzheimer's disease brain is catalyzed by Ca2+/calmodulin-dependent protein kinase II as demonstrated tandem mass spectrometry. Neurosci. Lett. 353, 185-188. doi: 10.1016/J.NEULET.2003. 09.037

Zaichick, S. V., McGrath, K. M., and Caraveo, G. (2017). The role of Ca $2+$ signaling in Parkinson's disease. Dis. Model. Mech. 10, 519-535. doi: 10.1242/ dmm. 028738

Conflict of Interest Statement: The authors declare that the research was conducted in the absence of any commercial or financial relationships that could be construed as a potential conflict of interest.

Copyright (c) 2018 Shimizu, Kanai, Sugawara, Uchida and Uchida. This is an openaccess article distributed under the terms of the Creative Commons Attribution License (CC BY). The use, distribution or reproduction in other forums is permitted, provided the original author(s) and the copyright owner(s) are credited and that the original publication in this journal is cited, in accordance with accepted academic practice. No use, distribution or reproduction is permitted which does not comply with these terms. 